\title{
Comparison of Outcomes of Oblique Lateral Interbody Fusion with Percutaneous Posterior Fixation in Lateral Position and Minimally Invasive Transforaminal Lumbar Interbody Fusion for Degenerative Spondylolisthesis
}

\author{
Yoshinao Koike ${ }^{1}$, Yoshihisa Kotani ${ }^{1,2}$, Hidemasa Terao ${ }^{1,3}$, Norimasa Iwasaki ${ }^{3}$ \\ ${ }^{1}$ Spine and Spinal Cord Center, Department of Orthopaedic Surgery, Steel Memorial Muroran Hospital, Muroran, Japan \\ ${ }^{2}$ Department of Orthopedic Surgery, Kansai Medical University Medical Center, Moriguchi, Japan \\ ${ }^{3}$ Department of Orthopedic Surgery, Hokkaido University Graduate School of Medicine, Sapporo, Japan
}

Study Design: Single-center retrospective study.

Purpose: To compare the physical function and quality of life (OOL) parameters of two minimally invasive surgical (MIS) procedures: oblique lateral interbody fusion with percutaneous posterior fixation in lateral position (OLIF-LPF) and minimally invasive transforaminal lumbar interbody fusion (MIS-TLIF) for single-level degenerative spondylolisthesis (DS).

Overview of Literature: To date, many options for the surgical treatment of lumbar DS and reports have described the effectiveness of minimally invasive lateral access surgery and MIS-TLIF. However, there is still a paucity of comparative data regarding the physical function and QOL outcomes of OLIF and MIS-TLIF.

Methods: Eighty-six patients were enrolled in this study (group 0: OLIF-LPF, n=38; group T: MIS-TLIF, n=48). We evaluated the operation time, estimated blood loss (EBL), postoperative laboratory data, preoperative and postoperative radiographic parameters, overall functional outcome with the Japanese Orthopedic Association Back Pain Evaluation Questionnaire (JOABPEQ) effectiveness rate, and Visual Analog Scale (VAS) score for low back pain, leg pain, and leg numbness.

Results: No statistical differences in operation time, EBL, and C-reactive protein level, 5 days postoperatively, between groups 0 and T. With respect to radiological outcome, preoperative and postoperative disc height change was significantly greater in group 0 than in group T (3.8 vs. $1.8 \mathrm{~mm}, p<0.05$ ). Both groups showed postoperative improvements in the clinical outcome scores of all JOABPEO domains, but the effectiveness rate increase in the psychological domain was significantly higher in group 0 than in group $\mathrm{T}$ (47.1\% vs. $14.6 \%, p<0.05)$. No differences in the preoperative and postoperative VAS score change were noted between the two groups in any of the items.

Received Oct 29, 2019; Revised Jan 5, 2020; Accepted Feb 18, 2020

Corresponding author: Yoshinao Koike

Spine and Spinal Cord Center, Department of Orthopaedic Surgery, Steel Memorial Muroran Hospital, 1-45, Chiribetsu-cho, Muroran, Hokkaido 050-0076, Japan

Tel: +81-143-44-4650, Fax: +81-143-47-0990, E-mail: yoshinao.koi@gmail.com

Co-corresponding author: Yoshihisa Kotani

Department of Orthopedic Surgery, Kansai Medical University Medical Center, 10-15, Fumizono-cho, Moriguchi, Osaka 570-8507, Japan

Tel: +81-6-6992-1001, Fax: +81-6-6992-4846, E-mail: yoshi96k@ja2.so-net.ne.jp 
Conclusions: The changes in physical function and QOL parameters after OLIF-LPF and MIS-TLIF were almost equivalent; however, OLIF-LPF had significant superiority in the psychological domain.

Keywords: Degenerative spondylolisthesis; Minimally invasive surgical procedures; Oblique lateral interbody fusion; Transforaminal lumbar interbody fusion

\section{Introduction}

Degenerative spondylolisthesis (DS) is common in individuals over 50 years, and patients often present with symptoms of spinal stenosis [1]. DS is commonly treated surgically with lumbar decompression and fusion [2]. Patients with symptomatic DS who have undergone surgery have significantly greater improvements in pain and function than those who have received nonoperative treatment and long-term follow-up [3].

To date, many options for the surgical treatment of lumbar DS have been reported [1-15]. One of these is posterior decompression and fusion $[2,4,5]$, and the superiority of minimally invasive surgery (MIS), such as minimally invasive transforaminal lumbar interbody fusion (MISTLIF), over traditional open procedures has often been reported [6-8]. Another option is minimally invasive lateral access surgery such as extreme lateral interbody fusion (XLIF) or oblique lateral interbody fusion (OLIF). These procedures use the minimally invasive, lateral retroperitoneal approach and have been recently used in the treatment of degenerative spine disorders including DS [9-15].

Further, reports have described the effectiveness of minimally invasive lateral access surgery and MIS-TLIF [9-13], and some preliminary studies have compared its clinical and radiological outcomes with XLIF $[9,14,15]$. However, there is still a paucity of comparative data regarding the physical function and quality of life (QOL) outcomes of OLIF and MIS-TLIF.

The objective of this study was to compare the changes in physical function and QOL parameters between OLIF and MIS-TLIF for single-level DS.

\section{Materials and Methods}

Between 2013 and 2017, we retrospectively reviewed 92 patients. The inclusion criteria for this study were the diagnosis of single-level DS that was treated with primary single-level minimally invasive lumbar interbody fusion surgery at Steel Memorial Muroran Hospital with a minimum of 1-year follow-up. Patients with multilevel DS and those who underwent posterior decompression surgery without fusion or traditional open TLIF procedures were excluded.

All procedures performed in this study were in accordance with the ethical standards of the institutional and/or national research committee (Steel Memorial Muroran Hospital Institutional Review Board approval no., J180603) and with the 1964 Helsinki Declaration and its later amendments or comparable ethical standards. Patients were informed that their data would be used for clinical research purposes and gave their consent.

Between 2013 and 2015, patients with symptomatic DS underwent MIS-TLIF. Our institution adopted OLIF in 2012 and the procedure had been improved. Between 2015 and 2017, patients with symptomatic DS mainly underwent OLIF with percutaneous posterior fixation in the lateral position (OLIF-LPF). Our normal clinical practice is to select MIS-TLIF for patients who require posterior direct decompression (e.g., patient with calcification or ossification of ligamentum flavum at the time of surgery); however, there was no such a case in this study. Group $\mathrm{O}$, had 42 patients who underwent OLIF-LPF, while 50 patients underwent MIS-TLIF and form group T. Each procedure was performed by one senior spine surgeon using the same procedure throughout. In groups $\mathrm{O}$ and $\mathrm{T}, 4$ (9.5\%) of 42 patients and $2(4 \%)$ of 50 , respectively, were lost to follow-up, therefore clinical information was available for 86 patients in total. 
For clinical evaluation, self-reporting with the Japanese Orthopedic Association Back Pain Evaluation Questionnaire (JOABPEQ) was used and the Visual Analog Scale (VAS), was used to assess low back pain, leg pain, and leg numbness. The questionnaires were administered before surgery and 1-year afterward. The JOABPEQ comprises five domains (low back pain, lumbar function, walking ability, social life function, and mental health) and has 25 questionnaire items with scores ranging from 0 to 100 points in each domain, with a higher score representing better function [16]. Using the JOABPEQ, a treatment is considered effective if the post-treatment score improves by $\geq 20$ points compared with the pretreatment score or if the pretreatment score is $<90$ points and the post-treatment score is $\geq 90$ points. The effectiveness rate was calculated as follows: (number of patients' treatment judged 'effective' $) /$ ( (total number of patients in the group)-(number of patients whose pre- and post-treatment scores are both $\geq 90)\}[16]$.

For radiological assessment, the disc height (DH) and slipping length (SL) were measured before surgery, immediately after surgery, 6 months after surgery, and at final follow-up using plain lateral radiographs in the lateral decubitus position (Fig. 1).

The degree of stenosis was evaluated with axial T2weighted magnetic resonance imaging (MRI) by Schizas's grading method: grade $\mathrm{A}$, no or minor stenosis; grade $\mathrm{B}$, moderate stenosis; grade $\mathrm{C}$, severe stenosis; and grade $\mathrm{D}$, extreme stenosis [17].

The invasiveness of surgery was evaluated based on operation time, estimated blood loss (EBL), and postoperative C-reactive protein (CRP) level.

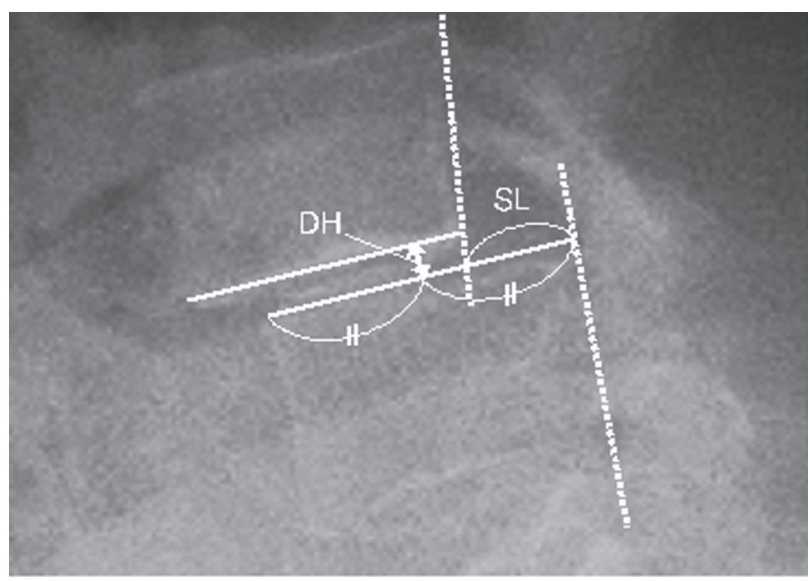

Fig. 1. Radiological parameters: DH and SL. DH, disc height; SL, slipping length.
Between-group differences in the preoperative background data, operation time, EBL, postoperative CRP level, radiological data, revision rate, JOABPEQ effectiveness rate, and VAS score were evaluated. The preoperative background data of the two surgical groups are shown in Table 1.

\section{Surgical procedure}

\section{1) OLIF-LPF}

In OLIF, the patient was placed in the right decubitus position. A transverse incision of approximately $4 \mathrm{~cm}$ was made on the skin in the left lateral region of the abdomen. After dissection of the external oblique, internal oblique, and transverse abdominal muscles, the retroperitoneal space was accessed by blunt dissection through the retroperitoneal fat tissue. The psoas muscle was retracted posteriorly. The targeted intervertebral disc space was exposed, and the tubular retractor system was set. Under direct visualization, a discectomy was performed and both cartilaginous endplates were thoroughly removed, and the polyetheretherketone (PEEK) cage with allograft was placed in the disc spaces. After performing OLIF, percutaneous screw insertion to the cephalad and caudal vertebrae was performed in the lateral position without position change using an intraoperative three-dimensional computed tomography (3D-CT)-based navigation system (O-arm; Medtronic, Minneapolis, MN, USA). We used the modified cortical bone trajectory (CBT) screw technique as previously reported (Fig. 2) [18]. Rods were then placed. This series of procedures were performed without intraoperative neuromonitoring. After the surgery, the patient was allowed to walk with a hard brace and was required to wear the brace for at least 2 months after the surgery (Fig. 3).

\section{2) MIS-TLIF}

In the MIS-TLIF procedure, the patient was placed in the prone position and a single longitudinal midline incision of approximately $4 \mathrm{~cm}$ was made. The bilateral paravertebral muscles were stripped and dissected to the inside of the facet joint to access the posterior column of the vertebral body. After expansion of the surgical field, modified CBT screw insertion (same as in the OLIF-LPF procedure) in the cephalad and caudal vertebrae was performed. For patients with severe spinal canal stenosis in addition to the intervertebral instability, adequate poste- 
Table 1. Comparison of background data between groups 0 and $T$

\begin{tabular}{|c|c|c|c|}
\hline Characteristic & Group 0 ( $n=38)$ & Group $T(n=48)$ & $p$-value \\
\hline Age (yr) & $72.1 \pm 11.4$ & $70.1 \pm 11.5$ & 0.444 \\
\hline Sex & & & 0.161 \\
\hline Male & $20(52.6)$ & $18(37.5)$ & \\
\hline Female & $18(47.4)$ & $30(62.5)$ & \\
\hline Degenerative spondylolisthesis level & & & 0.815 \\
\hline L3 & $5(13.2)$ & $6(12.5)$ & \\
\hline L4 & $33(86.8)$ & $42(87.5)$ & \\
\hline Body mass index $\left(\mathrm{kg} / \mathrm{m}^{2}\right)$ & $24.4 \pm 3.2$ & $23.7 \pm 5.1$ & 0.437 \\
\hline \multicolumn{4}{|l|}{ Comorbidity } \\
\hline Hypertension & $19(50.0)$ & $22(45.8)$ & 0.701 \\
\hline Ischemic heart disease & $0(0)$ & $3(6.3)$ & 0.329 \\
\hline Arrhythmia & $4(10.5)$ & $2(4.2)$ & 0.469 \\
\hline Pulmonary disease & $3(7.9)$ & $3(6.3)$ & 0.897 \\
\hline Chronic kidney disease & $13(34.2)$ & $23(47.9)$ & 0.201 \\
\hline Diabetes mellitus & $8(21.1)$ & $7(14.6)$ & 0.618 \\
\hline American Society of Anesthesiologists score & $2.0 \pm 0.4$ & $1.8 \pm 0.5$ & $0.010^{a)}$ \\
\hline \multicolumn{4}{|l|}{ Severity of stenosis } \\
\hline A & $11(28.9)$ & $14(29.2)$ & 0.947 \\
\hline B & $10(26.3)$ & $15(31.3)$ & \\
\hline C & $11(28.9)$ & $13(27.1)$ & \\
\hline $\mathrm{D}$ & $6(15.8)$ & $6(12.5)$ & \\
\hline \multicolumn{4}{|c|}{ Japanese Orthopedic Association Back Pain Evaluation Questionnaire } \\
\hline Low back pain & $34.0 \pm 29.6$ & $43.5 \pm 30.4$ & 0.148 \\
\hline Lumbar function & $50.5 \pm 27.1$ & $63.8 \pm 22.6$ & $0.030^{a)}$ \\
\hline Walking ability & $34.4 \pm 29.7$ & $41.0 \pm 27.3$ & 0.192 \\
\hline Social life & $43.0 \pm 20.3$ & $48.7 \pm 20.3$ & 0.060 \\
\hline Mental health & $40.3 \pm 17.5$ & $51.1 \pm 14.8$ & $0.002^{\text {al }}$ \\
\hline \multicolumn{4}{|l|}{ Visual Analog Scale } \\
\hline Low back pain & $63.9 \pm 24.3$ & $55.2 \pm 26.8$ & 0.147 \\
\hline Leg pain & $69.2 \pm 23.8$ & $59.9 \pm 29.5$ & 0.140 \\
\hline Leg numbness & $58.8 \pm 33.0$ & $59.6 \pm 32.0$ & 0.908 \\
\hline Follow-up (mo) & $18.1 \pm 8.5$ & $22.5 \pm 12.8$ & 0.069 \\
\hline
\end{tabular}

Values are presented as mean \pm standard deviation or number $(\%)$.

alIndicates statistically significant difference.

rior neural decompression was performed. Following unilateral facetectomy, the intervertebral disc was removed, both endplates were thoroughly decorticated, and a boomerang-shaped PEEK cage filled with local bone chips was inserted in the disc space. Rods were then placed, and the screw-rod construct was compressed to exert pressure on the interbody cage. This procedure was performed under intraoperative 3D-CT based navigation guidance. Af- ter surgery, the patient could walk with a hard brace and was required to wear the brace for at least 2 months after the surgery (Fig. 4).

\section{Statistical analysis}

Statistical comparisons of categorical variables between groups $\mathrm{O}$ and $\mathrm{T}$ were evaluated using only chi-square 

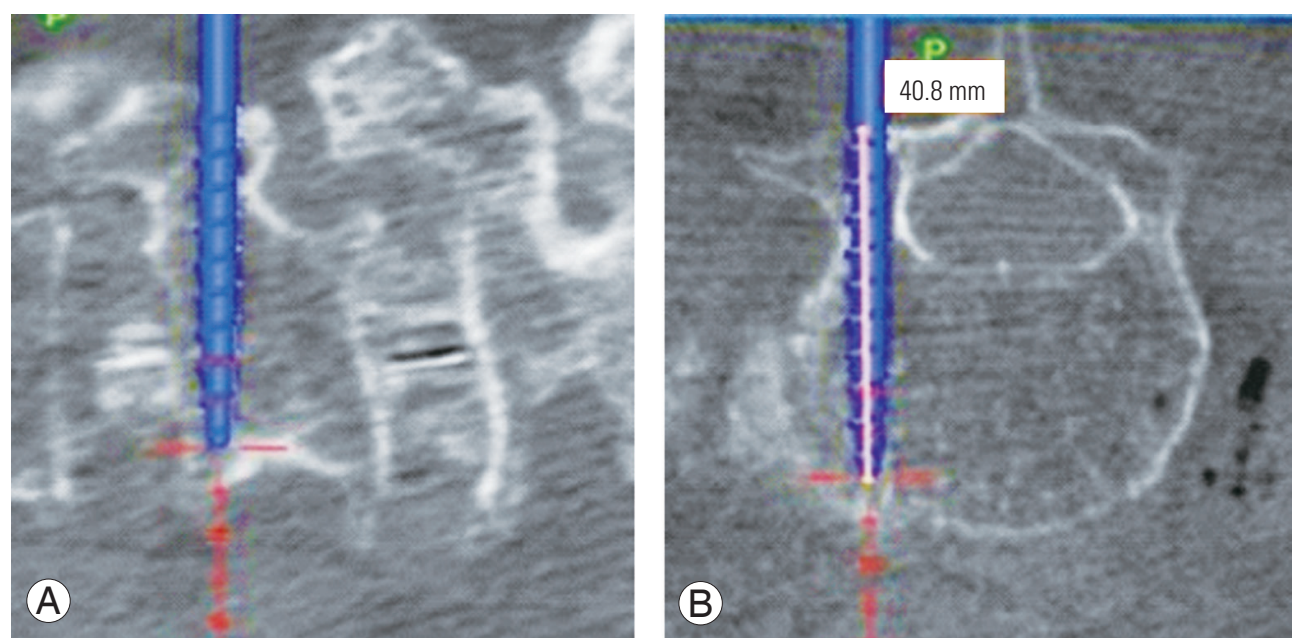

Fig. 2. Modified cortical bone trajectory screw method in our institution: (A) sagittal and (B) axial. The starting point is at the caudal border of the pedicle, and the trajectory takes a caudo-cephalad path almost parallel to the sagittal plane and more toward the anterior of the vertebral body than that of the original method.
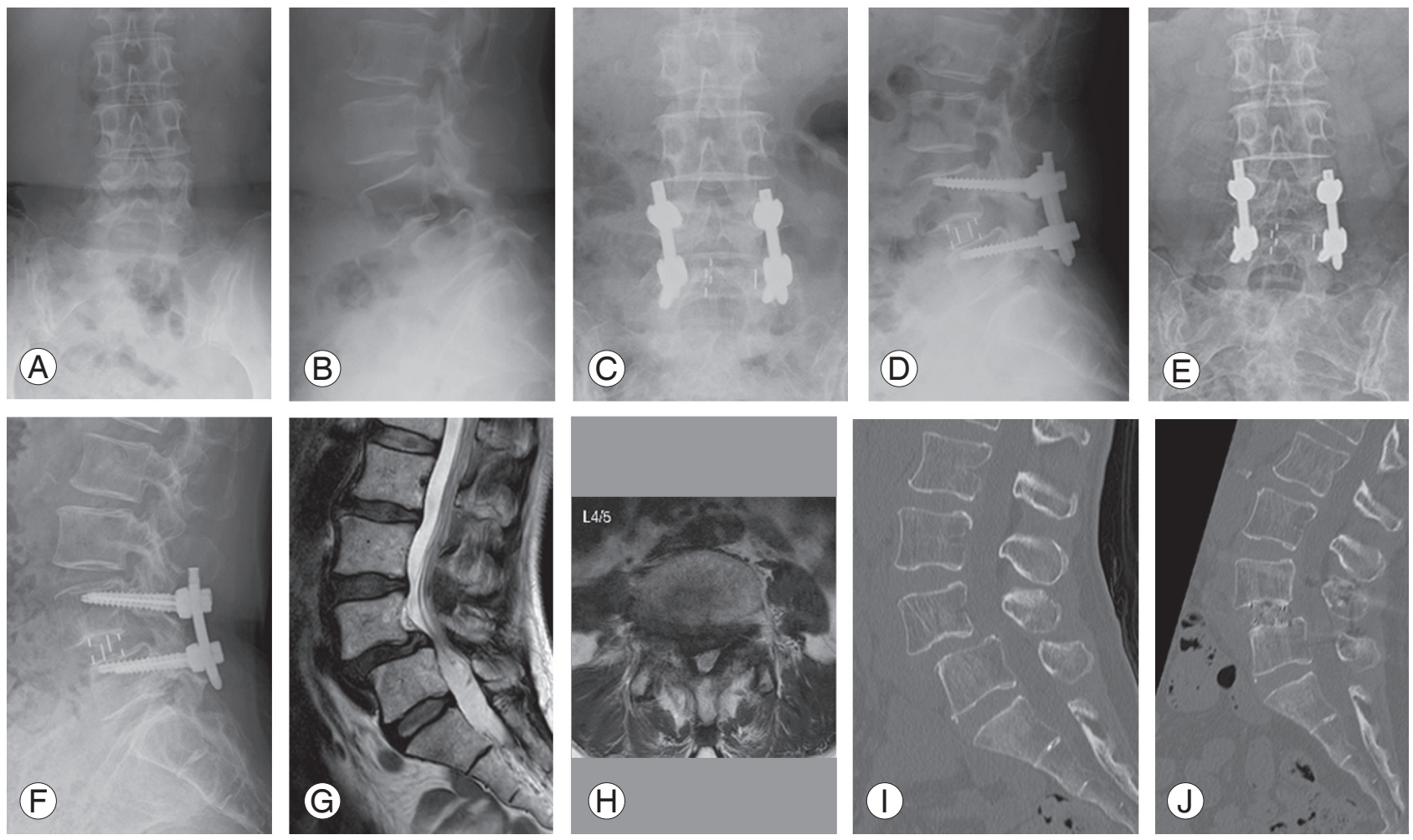

Fig. 3. A 54-year-old female patient with $L 4 / 5$ degenerative spondylolisthesis who underwent an oblique lateral interbody fusion with percutaneous posterior fixation in lateral position. (A-F) Plain radiograph: (A, B) preoperative; (C, D) 1 week after surgery; and (E, F) 1 year after surgery. (G, H) Preoperative T2-weighted magnetic resonance imaging. (I, J) Computed tomography: (I) preoperative and (J) 7 months after surgery.

tests, or chi-square tests with Yates' correction when the expected numbers were small. For continuous variables, differences between groups were evaluated with the twosample $t$-test. Differences in preoperative JOABPEQ scores between groups were evaluated with the Mann-
Whitney $U$-test, and differences in the JOABPEQ effectiveness rate were evaluated with test of population proportion. Data were analyzed using StatMate ver. 5.01 (ATMS Co. Ltd., Tokyo, Japan), with $p<0.05$ considered as statistically significant. 

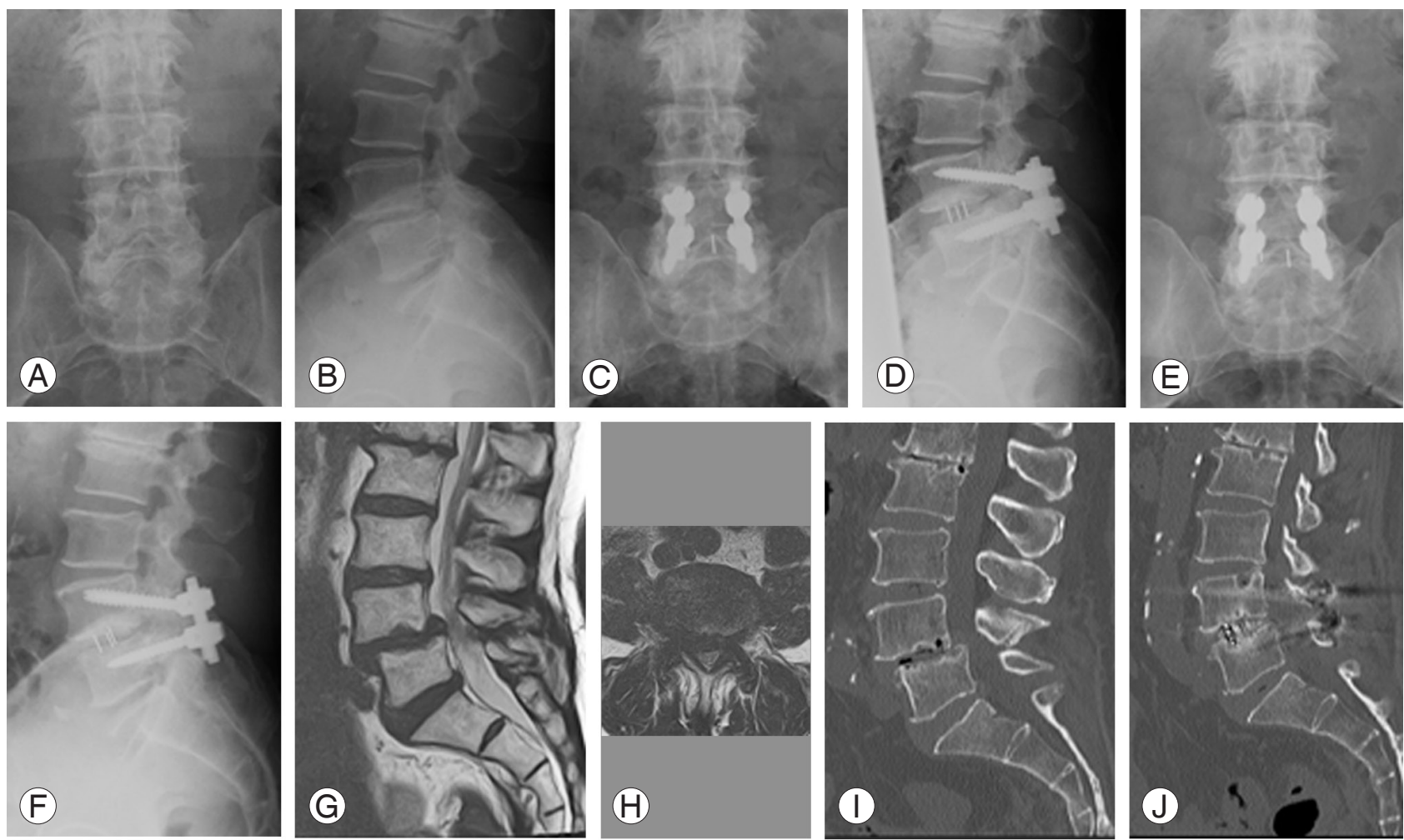

Fig. 4. A 72-year-old male patient with $L 4 / 5$ degenerative spondylolisthesis who underwent minimally invasive transforaminal lumbar interbody fusion. (A-F) Plain radiograph: (A, B) preoperative; (C, D) 1 week after surgery; and (E, F) 1 year after surgery. (G, H) Preoperative T2-weighted magnetic resonance imaging. (I, J) Computed tomography: (I) preoperative and (J) 7 months after surgery.

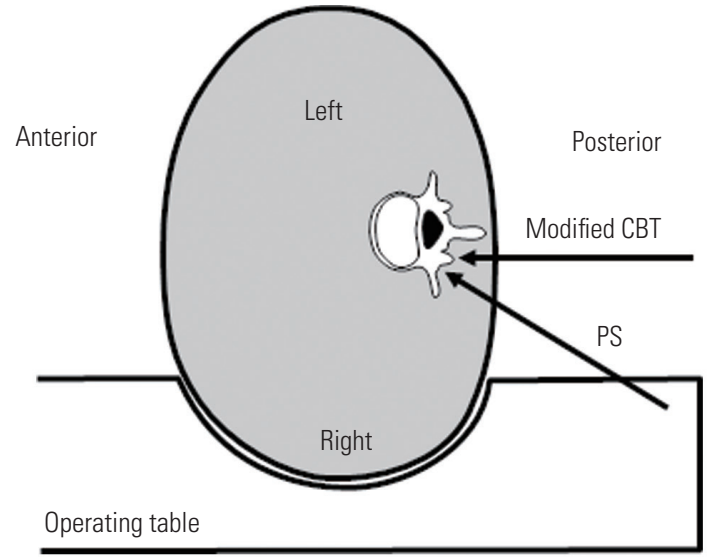

Fig. 5. In the lateral position, the working space for screw insertion on the right side is particularly limited. Modified CBT screws inserted parallel to the sagittal plane are easier to place than traditional pedicle screws. CBT, cortical bone trajectory.

\section{Results}

The mean follow-up period was 18.1 months (range, $12-$ 44 months) in group $\mathrm{O}$ and 22.5 months (range, 12-47 months) in group T. There were no statistical differences in terms of operation time or EBL between groups $\mathrm{O}$ and
$\mathrm{T}$ (Table 2). The postoperative CRP level was significantly higher in group $\mathrm{O}$ than in group $\mathrm{T}$ at 1 and 3 days after operation, but no difference was observed at 5 days after operation. In terms of radiological outcomes, the $\mathrm{DH}$ change was significantly greater in group $\mathrm{O}(3.8 \mathrm{~mm})$ than in group $\mathrm{T}(1.8 \mathrm{~mm})$, but no difference was observed for SL change. According to the time-series data of $\mathrm{DH}$ and SL, although DH and SL loss was observed postoperatively with time, no between-group difference was observed (Table 2). With respect to clinical outcomes, both groups showed improvement in all JOABPEQ domains postoperatively, but the effectiveness rate in the psychological domain was significantly higher in group $\mathrm{O}$ than in group $\mathrm{T}(p<0.05)$. In both groups, the postoperative VAS scores revealed significant improvement compared with the preoperative values in all of the items $(p<0.05)$ but no differences in the changes were noted between the two groups (Table 2). Revision surgery was required in four patients (10.5\%) and five patients (10.4\%) in groups $\mathrm{O}$ and $\mathrm{T}$, respectively, during the follow-up period. The summary of the revision cases is shown in Table 3. 
Table 2. Comparisons of surgical invasiveness, radiological data, and clinical outcomes between groups 0 and $\mathrm{T}$

\begin{tabular}{|c|c|c|c|}
\hline Variable & $\begin{array}{l}\text { Group } 0 \\
(n=38)\end{array}$ & $\begin{array}{l}\text { Group T } \\
(n=48)\end{array}$ & $p$-value \\
\hline Surgical time (min) & $111.9 \pm 23.6$ & $103.6 \pm 22.3$ & 0.096 \\
\hline Estimated blood loss (mL) & $51.7 \pm 37.8$ & $71.3 \pm 66.8$ & 0.091 \\
\hline \multicolumn{4}{|l|}{ C-reactive protein (mg/dL) } \\
\hline Preop & $0.11 \pm 0.11$ & $0.16 \pm 0.27$ & 0.274 \\
\hline Postop day 1 & $2.04 \pm 0.92$ & $1.08 \pm 0.63$ & $<0.001^{\mathrm{a})}$ \\
\hline Postop day 3 & $8.09 \pm 3.91$ & $4.49 \pm 3.11$ & $<0.001^{\text {a) }}$ \\
\hline Postop day 5 & $2.21 \pm 1.71$ & $2.34 \pm 1.67$ & 0.719 \\
\hline \multicolumn{4}{|l|}{$\mathrm{DH}$} \\
\hline Preop & $8.1 \pm 2.9$ & $8.8 \pm 2.6$ & 0.348 \\
\hline 1 wk & $11.9 \pm 2.0$ & $10.6 \pm 1.6$ & $<0.001^{a)}$ \\
\hline $6 \mathrm{mo}$ & $11.0 \pm 1.8$ & $9.5 \pm 1.5$ & $<0.001^{\text {a) }}$ \\
\hline Final follow-up & $10.4 \pm 1.8$ & $8.7 \pm 1.5$ & $<0.001^{\text {a) }}$ \\
\hline \multicolumn{4}{|l|}{ SL } \\
\hline Preop & $6.6 \pm 3.1$ & $7.4 \pm 2.6$ & 0.368 \\
\hline 1 wk & $3.6 \pm 2.8$ & $4.5 \pm 2.7$ & 0.149 \\
\hline $6 \mathrm{mo}$ & $3.8 \pm 2.8$ & $5.0 \pm 2.7$ & 0.066 \\
\hline Final follow-up & $3.9 \pm 2.9$ & $5.1 \pm 2.7$ & 0.069 \\
\hline \multicolumn{4}{|l|}{ DH change } \\
\hline Preop $\rightarrow 1$ wk & $3.8 \pm 2.5$ & $1.8 \pm 2.2$ & $<0.001^{\mathrm{a})}$ \\
\hline $1 \mathrm{wk} \rightarrow 6 \mathrm{mo}$ & $-1.0 \pm 1.2$ & $-1.2 \pm 1.0$ & 0.605 \\
\hline 1 wk $\rightarrow$ final follow-up & $-1.5 \pm 1.3$ & $-2.0 \pm 1.1$ & 0.112 \\
\hline \multicolumn{4}{|l|}{ SL change } \\
\hline Preop $\rightarrow 1$ wk & $-3.1 \pm 1.6$ & $-2.9 \pm 2.0$ & 0.462 \\
\hline $1 \mathrm{wk} \rightarrow 6 \mathrm{mo}$ & $0.3 \pm 0.4$ & $0.5 \pm 0.9$ & 0.103 \\
\hline $1 \mathrm{wk} \rightarrow$ final follow-up & $0.4 \pm 0.5$ & $0.6 \pm 1.0$ & 0.183 \\
\hline \multicolumn{4}{|c|}{ JOABPEQ effectiveness rate (\%) } \\
\hline Low back pain & 69.7 & 57.9 & 0.303 \\
\hline Lumbar function & 39.4 & 52.5 & 0.264 \\
\hline Walking ability & 65.6 & 65.8 & 0.988 \\
\hline Social life & 44.1 & 43.9 & 0.985 \\
\hline Mental health & 47.1 & 14.6 & $<0.001^{\mathrm{a})}$ \\
\hline \multicolumn{4}{|l|}{ VAS improvement } \\
\hline Low back pain & $32.1 \pm 31.3$ & $28.6 \pm 35.0$ & 0.647 \\
\hline Leg pain & $41.1 \pm 30.4$ & $27.9 \pm 41.8$ & 0.127 \\
\hline Leg numbness & $34.5 \pm 40.2$ & $38.2 \pm 40.6$ & 0.695 \\
\hline
\end{tabular}

Values are presented as mean \pm standard deviations or $\%$.

Preop, preoperative; Postop, postoperative; DH, disc height; SL, slipping length; JOABPEQ, Japanese Orthopedic Association Back Pain Evaluation Questionnaire; VAS, Visual Analog Scale.

a)Indicates statistically significant difference.

\section{Discussion}

The present study revealed that the improvements in physical function and QOL parameters of OLIF-LPF and MIS-TLIF for single-level DS were almost equivalent, but OLIF-LPF had significant superiority in the psychological domain. OLIF-LPF also had a shortened operation time and minimized surgical invasiveness.

The TLIF is an effective treatment method for DS, and many authors have reported its efficacy [4,5]. Various MIS-TLIF procedures have been developed [6-8,19-21], and their superiority to the traditional open procedure has often been reported [6-8]. Recently, minimally invasive lateral access surgeries such as OLIF and XLIF have attracted much attention [9-15], and our institution adopted OLIF in 2012. It allows direct access to the disc space, comprehensive disc space clearance, and placement of a large interbody graft. Many studies have reported its efficacy for the treatment of degenerative lumbar disease including DS [11-13].

In minimally invasive lateral access surgery, including OLIF, posterior direct decompression is generally not performed, as its ability to restore DH and indirectly decompress the neural elements improves neurological symptoms [22,23]. Fujibayashi et al. [23] analyzed 28 patients who underwent OLIF and measured the cross-sectional area of the thecal sac preoperatively and postoperatively using T2-weighted axial MRI. They reported that the spinal stenosis had been resolved by indirect decompression. In our institution, posterior direct decompression is not performed in OLIF, but as can be seen from our results, the change in $\mathrm{DH}$ was greater in group $\mathrm{O}$, and this decompression of the neural elements resulted in the improvement of the patients' physical function and QOL to approximately the same level as those of group $\mathrm{T}$. The reason for the superiority of OLIF-LPF in terms of the psychological domain of JOABPEQ is unknown, however, a possible reason might be that patients in group $\mathrm{T}$ were at a disadvantage for postoperative improvement in the psychological domain because of a significantly higher preoperative score.

OLIF was generally performed in the lateral position [11-13], and the extension of operative and anesthesia time caused by position change was one of the problems we faced in our institution after the introduction of OLIF. We, therefore, modified the surgical procedure and shortened the operative and anesthesia time by performing the 
Table 3. Summary of revision cases

\begin{tabular}{lccccccc} 
No. & Age $(\mathrm{yr})$ & Sex & Level of DS & Primary surgery & Time to revision (mo) & Reason for revision & Revision surgery \\
\hline 1 & 83 & M & L4 & L4-5 MIS-TLIF & 15 & L4-5 nonunion & L4-5 OLIF-LPF \\
\hline 2 & 83 & F & L4 & L4-5 MIS-TLIF & 12 & L3-4 ASD & L3-4 TLIF \\
\hline 3 & 76 & M & L3 & L3-4 MIS-TLIF & 26 & L2-3 ASD & L2-3 OLIF-AF \\
\hline 4 & 58 & F & L4 & L4-5 MIS-TLIF & 29 & L5-S1 ASD & L5-S1 TLIF \\
\hline 5 & 71 & F & L4 & L4-5 MIS-TLIF & 18 & L5-S1 ASD & L5-S1 TLIF \\
\hline 6 & 51 & F & L4 & L4-5 OLIF-LPF & 37 & L5-S1 ASD & L5-S1 OLIF-PF \\
\hline 7 & 67 & M & L4 & L4-5 OLIF-LPF & 0 & Screw deviation & Screw replacement \\
\hline 8 & 80 & F & L4 & L4-5 OLIF-LPF & 24 & L3-4 ASD & L3-4 OLIF-LPF \\
\hline 9 & 59 & M & L3 & L3-4 OLIF-LPF & 13 & L4-5 ASD & L4-5 OLIF-LPF \\
\hline
\end{tabular}

DS, degenerative spondylolisthesis; M, male; F, female; MIS, minimally invasive surgery; TLIF, transforaminal lumbar interbody fusion; OLIF-LPF, oblique lateral interbody fusion with percutaneous posterior fixation in lateral position; ASD, adjacent segment disease; AF, anterior fixation; PF, posterior fixation.

percutaneous screw insertion without a change in position. Furthermore, another advantage of OLIF-LPF is that patients could avoid the risks associated with being under anesthesia in the prone position. Blizzard and Thomas [24] analyzed 72 consecutive patients (300 screws) who underwent single-position lateral interbody fusion and percutaneous pedicle screw and rod placement and reported that the technique eliminated the prolonged time and extra staffing needed for intraoperative repositioning, which can reduce costs. However, screw insertion in the lateral position created several problems: the working space was limited with screw insertion on the right side (Fig. 5) and the fluoroscopy was difficult, with the time during operation tending to be prolonged which increased intraoperative radiation exposure. To solve these problems, we used modified CBT screws inserted parallel to the sagittal plane under an intraoperative 3D-CT-based navigation system [18]. In the present study, the CRP levels were significantly higher at 1 day and 3 days after operation in group $\mathrm{O}$ than in group T, but by 5 days after the operation, they were equivalent. Stopping the bleeding from the back muscle during posterior percutaneous screw placement is often difficult, and can cause a postoperative hematoma, which might cause elevation of the CRP in the early postoperative period.

Based on the results of the present study, both OLIFLPF and MIS-TLIF are effective surgical methods for lumbar DS. In our institution, when performing MIS-TLIF, a series of surgical procedures were performed with the same single incision without making multiple small skin incisions for percutaneous screw insertion because the posterior decompression area was adjacent to the modi- fied CBT screw insertion points. The midline skin incision was, therefore, a little longer than is usually reported in the published literature [6-8]. As was recently reported by several authors, if the endoscopy technique is simultaneously used for posterior decompression and interbody fusion, less invasive surgery may be possible [19-21]. In OLIF, posterior percutaneous screw insertion is necessary to keep the fused vertebrae stable until bone union. In a previous report, Tempel et al. [25] noted the correlation between graft subsidence and the need for revision surgery in patients after stand-alone lateral lumbar interbody fusion and recommended the placement of supplementary posterior instrumentation at the time of initial surgery in these patients if they have an increased risk of graft subsidence [25]. In the future, less invasive lateral access interbody fusion may be possible if stable postoperative results are achieved with new surgical devices such as the interbody cage equipped with built-in screws, or the lowprofile plate system for anterior vertebral fusion. These two surgical procedures are likely to be further improved because of the increasing demand for MIS in our aging society.

There were some limitations to this study. First, the patients of group T mainly underwent surgery from 2013 to 2015 whereas those of group $\mathrm{O}$ were treated during 2015-2017. Although there were no significant differences in terms of the follow-up period between the two groups, several statistical differences were detected in their background factors, and a properly designed randomized trial is needed to compare the effectiveness of the two MIS procedures more accurately. Second, the frequency of postoperative evaluation with JOABPEQ was low, and we only 
evaluated patient outcomes at 1 year after surgery. There may have been early differences in patient outcomes and further studies that include evaluation during the early postoperative period are needed. Third, several important spinal radiographic parameters (e.g., spinopelvic parameters and sagittal spinal balance) were not measured, because preoperative and postoperative whole spine radiographs were not taken in many cases in this study. Future evaluation of the effect of each MIS procedure on these spinal radiographic parameters is warranted.

\section{Conclusions}

The changes in physical function and QOL parameters of OLIF-LPF and MIS-TLIF for single-level DS were almost equivalent, but OLIF-LPF had significant superiority in the psychological domain. OLIF-LPF had a shortened operation time and less surgical invasiveness, which were equivalent to those of MIS-TLIF. These findings suggest that OLIF-LPF is an effective and less invasive treatment method for DS that is comparable with MIS-TLIF.

\section{Conflict of Interest}

No potential conflict of interest relevant to this article was reported.

\section{Author Contributions}

Conception and design, data acquisition, and drafting of the manuscript: Koike Y, Kotani Y; analysis of data: Koike $\mathrm{Y}$; and critical revision and supervision: Terao $\mathrm{H}$, Iwasaki $\mathrm{N}$.

\section{References}

1. Sengupta DK, Herkowitz HN. Degenerative spondylolisthesis: review of current trends and controversies. Spine (Phila Pa 1976) 2005;30(6 Suppl):S71-81.

2. Weinstein JN, Lurie JD, Olson PR, Bronner KK, Fisher ES. United States' trends and regional variations in lumbar spine surgery: 1992-2003. Spine (Phila Pa 1976) 2006;31:2707-14.

3. Abdu WA, Sacks OA, Tosteson AN, et al. Long-term results of surgery compared with nonoperative treatment for lumbar degenerative spondylolisthesis in the Spine Patient Outcomes Research Trial (SPORT).
Spine (Phila Pa 1976) 2018;43:1619-30.

4. Adogwa O, Parker SL, Davis BJ, et al. Cost-effectiveness of transforaminal lumbar interbody fusion for grade I degenerative spondylolisthesis. J Neurosurg Spine 2011;15:138-43.

5. Xu H, Tang H, Li Z. Surgical treatment of adult degenerative spondylolisthesis by instrumented transforaminal lumbar interbody fusion in the Han nationality. J Neurosurg Spine 2009;10:496-9.

6. Dhall SS, Wang MY, Mummaneni PV. Clinical and radiographic comparison of mini-open transforaminal lumbar interbody fusion with open transforaminal lumbar interbody fusion in 42 patients with longterm follow-up. J Neurosurg Spine 2008;9:560-5.

7. Parker SL, Mendenhall SK, Shau DN, et al. Minimally invasive versus open transforaminal lumbar interbody fusion for degenerative spondylolisthesis: comparative effectiveness and cost-utility analysis. World Neurosurg 2014;82:230-8.

8. Price JP, Dawson JM, Schwender JD, Schellhas KP. Clinical and radiologic comparison of minimally invasive surgery with traditional open transforaminal lumbar interbody fusion: a review of 452 patients from a single center. Clin Spine Surg 2018;31:E121-6.

9. Isaacs RE, Sembrano JN, Tohmeh AG; SOLAS Degenerative Study Group. Two-year comparative outcomes of MIS lateral and MIS transforaminal interbody fusion in the treatment of degenerative spondylolisthesis: part II: radiographic findings. Spine (Phila Pa 1976) 2016;41 Suppl 8:S133-44.

10. Ozgur BM, Aryan HE, Pimenta L, Taylor WR. Extreme lateral interbody fusion (XLIF): a novel surgical technique for anterior lumbar interbody fusion. Spine J 2006;6:435-43.

11. Jin C, Jaiswal MS, Jeun SS, Ryu KS, Hur JW, Kim JS. Outcomes of oblique lateral interbody fusion for degenerative lumbar disease in patients under or over 65 years of age. J Orthop Surg Res 2018;13:38.

12. Ohtori S, Orita S, Yamauchi K, et al. Mini-open anterior retroperitoneal lumbar interbody fusion: oblique lateral interbody fusion for lumbar spinal degeneration disease. Yonsei Med J 2015;56:1051-9.

13. Sato J, Ohtori S, Orita S, et al. Radiographic evaluation of indirect decompression of mini-open anterior retroperitoneal lumbar interbody fusion: oblique lateral interbody fusion for degenerated lumbar spondylolisthesis. Eur Spine J 2017;26:671-8. 
14. Kono Y, Gen H, Sakuma Y, Koshika Y. Comparison of clinical and radiologic results of mini-open transforaminal lumbar interbody fusion and extreme lateral interbody fusion indirect decompression for degenerative lumbar spondylolisthesis. Asian Spine J 2018;12:356-64.

15. Sembrano JN, Tohmeh A, Isaacs R; SOLAS Degenerative Study Group. Two-year comparative outcomes of MIS lateral and MIS transforaminal interbody fusion in the treatment of degenerative spondylolisthesis: part I: clinical findings. Spine (Phila Pa 1976) 2016;41 Suppl 8:S123-32.

16. Fukui M, Chiba K, Kawakami M, et al. JOA Back Pain Evaluation Questionnaire (JOABPEQ)/JOA Cervical Myelopathy Evaluation Questionnaire (JOACMEQ): the report on the development of revised versions. April 16, 2007. The Subcommittee of the Clinical Outcome Committee of the Japanese Orthopaedic Association on Low Back Pain and Cervical Myelopathy Evaluation. J Orthop Sci 2009;14:34865.

17. Schizas C, Theumann N, Burn A, et al. Qualitative grading of severity of lumbar spinal stenosis based on the morphology of the dural sac on magnetic resonance images. Spine (Phila Pa 1976) 2010;35:191924.

18. Elmekaty M, Kotani Y, Mehy EE, et al. Clinical and radiological comparison between three different minimally invasive surgical fusion techniques for single-level lumbar isthmic and degenerative spondylolisthesis: minimally invasive surgical posterolateral fusion versus minimally invasive surgical transforaminal lumbar interbody fusion versus midline lumbar fusion. Asian Spine J 2018;12:870-9.
19. He EX, Guo J, Ling QJ, Yin ZX, Wang Y, Li M. Application of a narrow-surface cage in full endoscopic minimally invasive transforaminal lumbar interbody fusion. Int J Surg 2017;42:83-9.

20. Heo DH, Son SK, Eum JH, Park CK. Fully endoscopic lumbar interbody fusion using a percutaneous unilateral biportal endoscopic technique: technical note and preliminary clinical results. Neurosurg Focus 2017;43:E8.

21. Zhang Y, Xu C, Zhou Y, Huang B. Minimally invasive computer navigation-assisted endoscopic transforaminal interbody fusion with bilateral decompression via a unilateral approach: initial clinical experience at one-year follow-up. World Neurosurg 2017;106:291-9.

22. Oliveira L, Marchi L, Coutinho E, Pimenta L. A radiographic assessment of the ability of the extreme lateral interbody fusion procedure to indirectly decompress the neural elements. Spine (Phila Pa 1976). 2010;35(26 Suppl):S331-7.

23. Fujibayashi S, Hynes RA, Otsuki B, Kimura H, Takemoto M, Matsuda S. Effect of indirect neural decompression through oblique lateral interbody fusion for degenerative lumbar disease. Spine (Phila Pa 1976) 2015;40:E175-82.

24. Blizzard DJ, Thomas JA. MIS single-position lateral and oblique lateral lumbar interbody fusion and bilateral pedicle screw fixation: feasibility and perioperative results. Spine (Phila Pa 1976) 2018;43:440-6.

25. Tempel ZJ, McDowell MM, Panczykowski DM, et al. Graft subsidence as a predictor of revision surgery following stand-alone lateral lumbar interbody fusion. J Neurosurg Spine 2018;28:50-6. 